

Nanophotonics.SPIEDigitalLibrary.org

\title{
Review of optical detection of single molecules beyond the diffraction and diffusion limit using plasmonic nanostructures
}

Farzia Karim

Todd B. Smith

Chenglong Zhao 


\title{
Review of optical detection of single molecules beyond the diffraction and diffusion limit using plasmonic nanostructures
}

\author{
Farzia Karim, ${ }^{\mathrm{a}}$ Todd B. Smith, ${ }^{\mathrm{b}}$ and Chenglong Zhao ${ }^{\mathrm{a}, \mathrm{b}, *}$ \\ ${ }^{a}$ University of Dayton, Department of Electro-Optics and Photonics, Dayton, \\ Ohio, United States \\ ${ }^{b}$ University of Dayton, Department of Physics, Dayton, Ohio, United States
}

\begin{abstract}
Single-molecule detection has become a unique and indispensable tool for the study of molecular motions and interactions at the single-molecule level. Unlike ensemble measurement where the information is averaged, single-molecule analysis yields invaluable information on both the individual molecular properties and their microenvironment. Among the various technologies for the detection of single molecules, the detection with optical methods has many advantages in terms of its high sensitivity, electrical passiveness, and robustness. The recent advances in the engineering of either the excitation light or the solution of the molecules have paved the way for enhanced single-molecule detection. We present recent developments and future perspectives for single-molecule detection in the following three regimes: on a dry surface, in solutions at ultralow concentrations, and in solutions at native physiological concentrations. (C) The Authors. Published by SPIE under a Creative Commons Attribution 3.0 Unported License. Distribution or reproduction of this work in whole or in part requires full attribution of the original publication, including its DOI. [DOI: 10.1117/1.JNP.12.012504]
\end{abstract}

Keywords: single-molecule detection; optical nanoantennas; plasmonic nanostructures.

Paper 17049SSV received Apr. 19, 2017; accepted for publication Jun. 5, 2017; published online Jun. 22, 2017.

\section{Introduction}

The goal of sensing in biology is to have the capability to study a wide variety of single-molecule behaviors. Optical single-molecule detection is one such technology that enables the study of molecular motion and interaction at the single-molecule level, and it has become an indispensable tool in many applications, such as diagnostics, ${ }^{1}$ DNA sequencing, ${ }^{2,3}$ and molecular biology. ${ }^{4}$ Single-molecule analysis yields invaluable information on both the individual molecular properties and their microenvironment, unlike the information that is normally disguised in ensemble measurements.

Among the various single-molecule technologies, the detection with optical methods stands out because of its advantages, such as greater sensitivity, electrical passiveness, and robustness. Optical detection of single molecules can also be used over a wide range of concentrations. For simplicity, this review mainly focuses on the single-molecule detection in the following three regimes: (1) on a dry surface, (2) in solutions at ultralow concentrations, and (3) in solutions at native physiological concentrations. Optical detection of single molecules on a dry surface can be enhanced using nanostructures that enhance the fluorescence and modify the radiation direction. The detection in solutions at ultralow concentrations requires the development of ultrasensitive technology to distinguish the few molecules in the solution. A sample solution is at an ultralow concentration typically because either the sample itself is rare and hard to prepare in high concentrations or it is purposely diluted to facilitate single-molecule detection. The optical detection of single molecules in solutions at native physiological concentrations is very challenging but highly desired because meaningful information for many biologically relevant processes can be obtained only under native physiological conditions. ${ }^{5,6}$

*Address all correspondence to: Chenglong Zhao, E-mail: czhao1@udayton.edu 
With the rapid development of nanotechnology, both the excitation light and the solution of the molecules can be properly engineered to enhance single-molecule detection in all three regimes. For example, plasmonic nanostructures, which consist of carefully designed metallic nanostructures, have been used to enhance the single-molecule detection at both ultralow and high concentrations. ${ }^{7-12}$ Another easy-to-ignore but critically important factor for singlemolecule detection is the time required for a successful detection. Due to the small size of the active detection area, it can take an impractically long time for the molecules to diffuse into the detection area, especially at ultralow concentrations. Fortunately, several methods have been proposed to solve this problem as will be discussed in this article.

The remainder of this review article is outlined as follows: Sec. 2 will describe the techniques for the advanced detection of single molecules on a surface and at low concentrations; Sec. 3 will introduce methods that have been applied to overcome the diffusion limit or the detection-time issue; Sec. 4 will review the recent developments for the optical detection of single molecules at native physiological concentrations; and finally, Sec. 5 will conclude with a discussion of the perspectives for future developments in this promising field.

\section{Enhanced Single-Molecule Detection on a Surface and at Low Concentrations}

In the past few decades, engineering of near-field radiation with plasmonic nanostructures has proven to be a promising pathway for enhancing the weak signals from molecules with low quantum yields. Plasmonics is a research field that investigates the interaction of electromagnetic waves with metallic nanostructures. ${ }^{13,14}$ In recent years, the study of plasmonics has been extended to many nonmetallic materials, such as doped semiconductor nanoparticles ${ }^{15,16}$ and graphene. ${ }^{17,18}$ The surface plasmon resonance (SPR) on these nanostructures, which is a condition in which the electromagnetic wave resonantly couples with the free-electron motion of the nanostructures, can focus light far beyond the diffraction limit. ${ }^{19-21}$ Hundreds of such nanostructures can be fabricated on a substrate with each nanostructure serving as an independent nanodetector for the enhanced single-molecule detection. Therefore, it enables label-free and parallel detection of single molecules with significantly improved throughput. It also allows the enhancement of the fluorescence of molecules near these plasmonic nanostructures. The fluorescence enhancement $(\mathrm{FE})$ factor is defined as ${ }^{22,23}$

$$
\mathrm{FE}=\frac{\gamma_{\mathrm{exc}}}{\gamma_{\mathrm{exc}}^{o}} \frac{q}{q^{o}} \frac{\eta_{c}}{\eta_{c}^{o}},
$$

where $\gamma_{\mathrm{exc}} / \gamma_{\mathrm{exc}}^{0}$ gives the enhancement of the excitation rate that is directly related to the field enhancement of the plasmonic nanostructures, $q / q^{o}$ indicates the enhancement of the quantum yield of the molecules due to the presence of the plasmonic nanostructures, and $\eta_{c} / \eta_{c}^{o}$ represents the enhancement of the collection efficiency in the presence of the plasmonic nanostructures. The superscript o indicates the corresponding quantities in free space.

A wide variety of plasmonic nanostructures, also called optical nanoantennas because of their similarity to conventional radio frequency (RF) antennas, have been developed for singlemolecule detection. ${ }^{24-26}$ The most simple and easy-to-realize optical nanoantenna is a spherical nanoparticle, which has been used to reduce the observation volume by four orders of magnitude beyond the diffraction limit. ${ }^{27}$ Similar to an RF dipole antenna, an optical dipole nanoantenna $(\mathrm{ODN})$, which consists of a metallic nanorod (NR), has a response to the excitation light that strongly depends on its aspect ratio and can be carefully designed to optimize the excitation and emission rate for single-molecule detection. Figure 1(a) shows such an ODN for the detection of light-harvesting complex 2 (LH2). The ODN consists of a gold nanorod (AuNR) on a glass coverslip and can be prepared in arrays for parallel detection as shown in the inset of Fig. 1(a). The study of individual LH2 at ambient conditions without the help of optical nanoantennas is limited by the low fluorescence efficiency and its limited photostability. With the help of the ODN, an FE of light from the LH2 with more than a 500-fold enhancement has been demonstrated at the single-molecule level. ${ }^{24}$ Figure 1 (b) shows the fluorescence image of the 
(a)



(c)

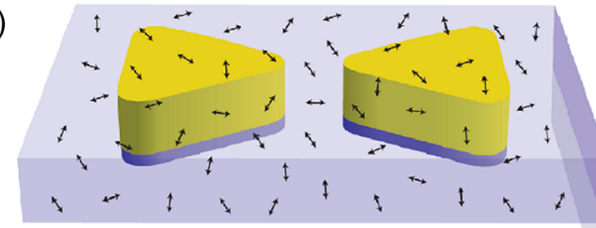

(d)

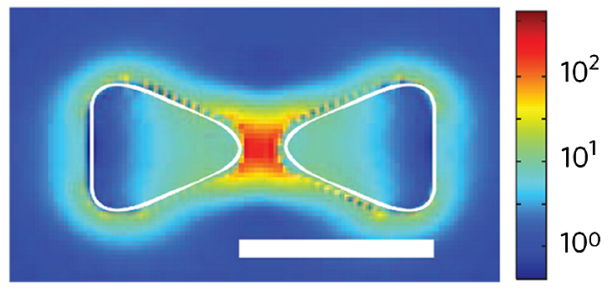

(b)

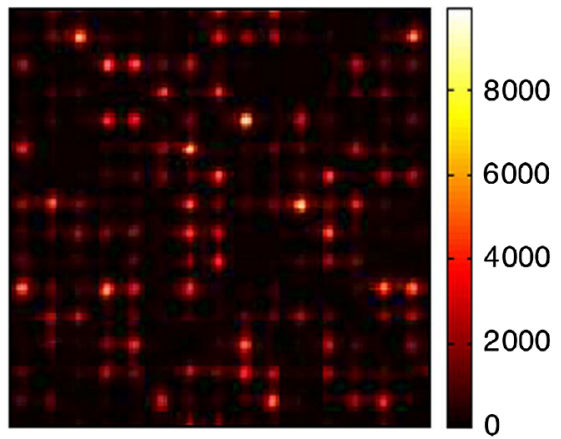

(e)



Fig. 1 (a) Schematic representation of an AuNR surrounded with LH2 complex. Inset: schematic of NRs with increasing length. (b) Fluorescence images of LH2 coated over an array of $160 \mathrm{~nm}$ AuNRs. Reproduced with permission from Ref. 24. (c) Schematic of bowtie nanoantenna (gold) coated with $\mathrm{N}, \mathrm{N}^{\prime}$-bis (2,6-diisopropylphenyl)-1,6,11,16-tetra-[4-(1,1,3,3-tetramethylbutyl) phenoxy] quaterrylene-3,4:13,14-bis (dicarboximide) (TPQDI) molecules (black arrows) in Poly (methyl methacrylate) (PMMA) (light blue) on a transparent substrate. (d) Finite-difference time-domain calculation of local intensity enhancement. Scale bar, $100 \mathrm{~nm}$. (e) Confocal scan of 16-bowtie nanoantennas coated with TPQDI molecules at high concentrations. Scale bar, $4 \mu \mathrm{m}$. (f) Fluorescence time trace of a single TPQDI/PMMA-coated bowtie nanoantenna. Reproduced with permission from Ref. 25.

LH2 on an ODN array. Interestingly, the authors have claimed the first demonstration of photon antibunching from a single LH complex under ambient conditions, which shows its potential application as a quantum emitter. The field enhancement, which is defined as the ratio between the magnitudes of the electric field near the optical nanoantenna to that of the incident light, is limited for a single ODN.

To increase the field enhancement, a similar optical nanoantenna can be put together to form a small gap. The near-field light coupling between the adjacent nanoantennas can give rise to a large field enhancement in the gap. Figure 1(c) shows such a coupled optical nanoantenna in a bowtie shape, a so-called bowtie nanoantenna. In addition to the huge field enhancement in the gap of the bowtie nanoantenna as shown in the simulation in Fig. 1(d), another advantage lies in its broadband response. Kinkhabwala et al. ${ }^{25}$ demonstrated large single-molecule FE by the bowtie nanoantenna. The bowtie nanoantennas are fabricated on a quartz coverslip with electronbeam lithography, and the low-quantum-efficiency fluorescent dye TPQDI is doped in PMMA and spin coated on the bowtie nanoantennas as shown in Fig. 1(c). The fluorescence of lowquantum-efficiency emitters has been shown to have a much higher potential to be enhanced in the presence of the optical nanoantennas than high-quantum-efficiency emitters. ${ }^{28,29}$ Figure $1(\mathrm{e})$ shows the experimental measurement of the FE of single molecules on 16 bowtie nanoantennas. Figure 1(f) shows the fluorescence time trace of a single molecule at one bowtie nanoantenna. A FE factor as high as 1340 has been reported for these nanoantennas.

The FE of a molecule in the gap of an optical nanoantenna strongly depends on the gap size of the optical nanoantenna. To further enhance the single-molecule detection, optical 
nanoantennas with gap sizes in the sub-10 nm range are desired. However, it is not an easy task to fabricate such small gap sizes with top-down nanofabrication methods, such as electron-beam lithography or focused ion-beam milling. To solve this problem, a simple yet clever design is proposed as shown in Fig. 2(a). In this system, a silver nanocube sits on top of a dielectric layer that is sandwiched between the nanocube and a gold film, which is a so-called nanoscale patch antenna (NPA). ${ }^{30,33-36}$ The gap of this NPA can be precisely controlled in the range of 5 to $15 \mathrm{~nm}$ during the spin-coating process used to fabricate the dielectric layer. ${ }^{30}$ Single fluorescent emitters are embedded in the dielectric layer. Figure 2(b) shows the fundamental plasmonic mode of NPA together with the large field enhancement in the gap. An enhanced spontaneous emission rate that exceeds 1000 has been obtained in the measurements of fluorescence lifetimes of different ensembles of emitters. The NPA also facilitates directional emission of the single molecules and therefore improves the collection efficiency of the system. ${ }^{30}$

In addition to top-down nanofabrication methods that are commonly used to fabricate optical nanoantennas for enhanced single-molecule detection, metallic nanoparticles, such as gold and silver nanoparticles, can be synthesized with bottom-up methods and assembled into well-organized nanostructures with extremely small gaps for enhanced single-molecule detection. For example, the self-assembly of metallic nanoparticles using the DNA origami technique has been extensively used to build plasmonic nanostructures for the detection of single molecules. ${ }^{37-41}$ The nanogaps in these nanostructures also allow for surface-enhanced Raman scattering (SERS) detection with single-molecule sensitivity. ${ }^{42}$ Enhancement factors in the range from $2.8 \times 10^{4}$ to $>1 \times 10^{10}$ have been reported in the literature. ${ }^{42-46}$ However, the reproducibility of the SERS sites is still poor, which results in unquantifiable SERS signals. To overcome these limitations, Lim et al. ${ }^{31}$ demonstrated a new form of nanostructure that is built with DNA-modified gold nanoparticles known as gold nanobridged nanogap particles (Au-NNPs) as shown in Fig. 2(e). Uniform $1 \mathrm{~nm}$ gaps can be formed between the gold core and the gold shell, and Raman dyes can be precisely loaded into the gap. Therefore, highly stable and quantitative SERS signals can be generated and amplified by roughly two orders of magnitude on this Au-NNP. Figures 2(f) and 2(g) show the distribution of the SERS enhancement factor of the Raman spectrum of Au-NNPs at 1190 and $1580 \mathrm{~cm}^{-1}$, respectively.

The aforementioned methods can generate significantly enhanced Raman signals for singlemolecule detection; however, the SERS site is typically fixed on the substrate making it hard to create an SERS map, which contains valuable positional information. Tip-enhanced Raman scattering (TERS) has been developed not only for detection but also for chemical recognition of individual molecules at any desired location on a surface. ${ }^{47,48}$ Figure $2(\mathrm{~h})$ schematically shows a typical TERS configuration, which consists of a gap formed between a scanning tip and a metallic surface. A submonolayer of molecules is immobilized on the metallic surface, and the scanning tip can freely move over the surface to create an SERS map of the immobilized molecules. This special configuration offers stable control of the gaps at incredibly small tipsample distances. It also provides in situ tuning of the plasmon resonance of the nanocavity, which is defined by the tip and substrate. ${ }^{49}$ Recently, Jiang et al. ${ }^{32}$ have shown the ability to distinguish adjacent molecules $\left(\mathrm{H}_{2}\right.$ TBPP and $\left.\mathrm{ZnTPP}\right)$ in real space that are within the van der Waals contact distance by using this method. Figure 2(i) shows that both ZnTPP and $\mathrm{H}_{2}$ TBPP molecules are coadsorbed at the step edge with a total of six molecules arranged alternately due to the van der Waals interactions.

In addition to the aforementioned techniques, Förster resonance energy transfer (FRET) is another important method for single-molecule measurement. FRET depends on the nonradiative energy transfer from a donor fluorophore to an acceptor fluorophore, which is distance dependent. Therefore, it allows for the measurement of molecular distances at nanometer scales. FRET can also be enhanced using plasmonic nanostructures. For example, Zhang et al. ${ }^{50}$ have investigated the effect of a metallic silver nanoparticle on FRET between a nearby donoracceptor pair and showed enhanced FRET efficiency due to the metal nanoparticles. Moreover, the resonance energy transfer can directly occur between plasmonic nanostructures and dye molecules. ${ }^{51}$ For example, Zheng's group has reported plasmon-induced resonance energy transfer from single AuNRs to merocyanine dye molecules. ${ }^{52}$ This shows that AuNRs can function as donors that have a larger absorption cross section than the dye molecules, which leads to an enhanced acceptor excitation. 

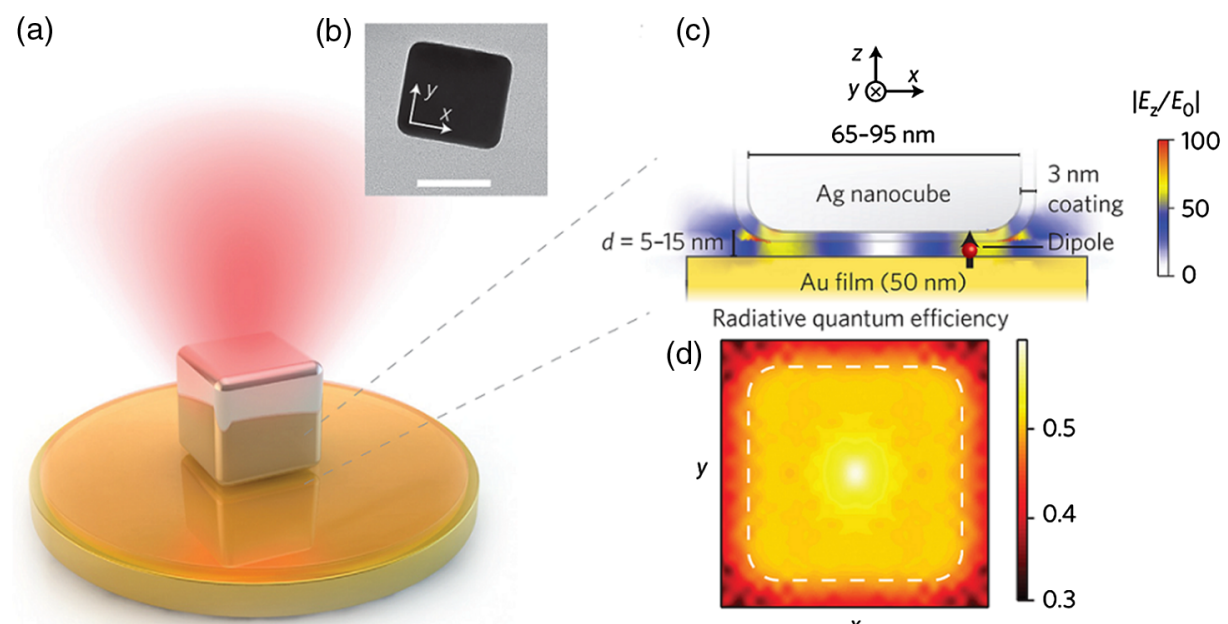

Radiative quantum efficiency
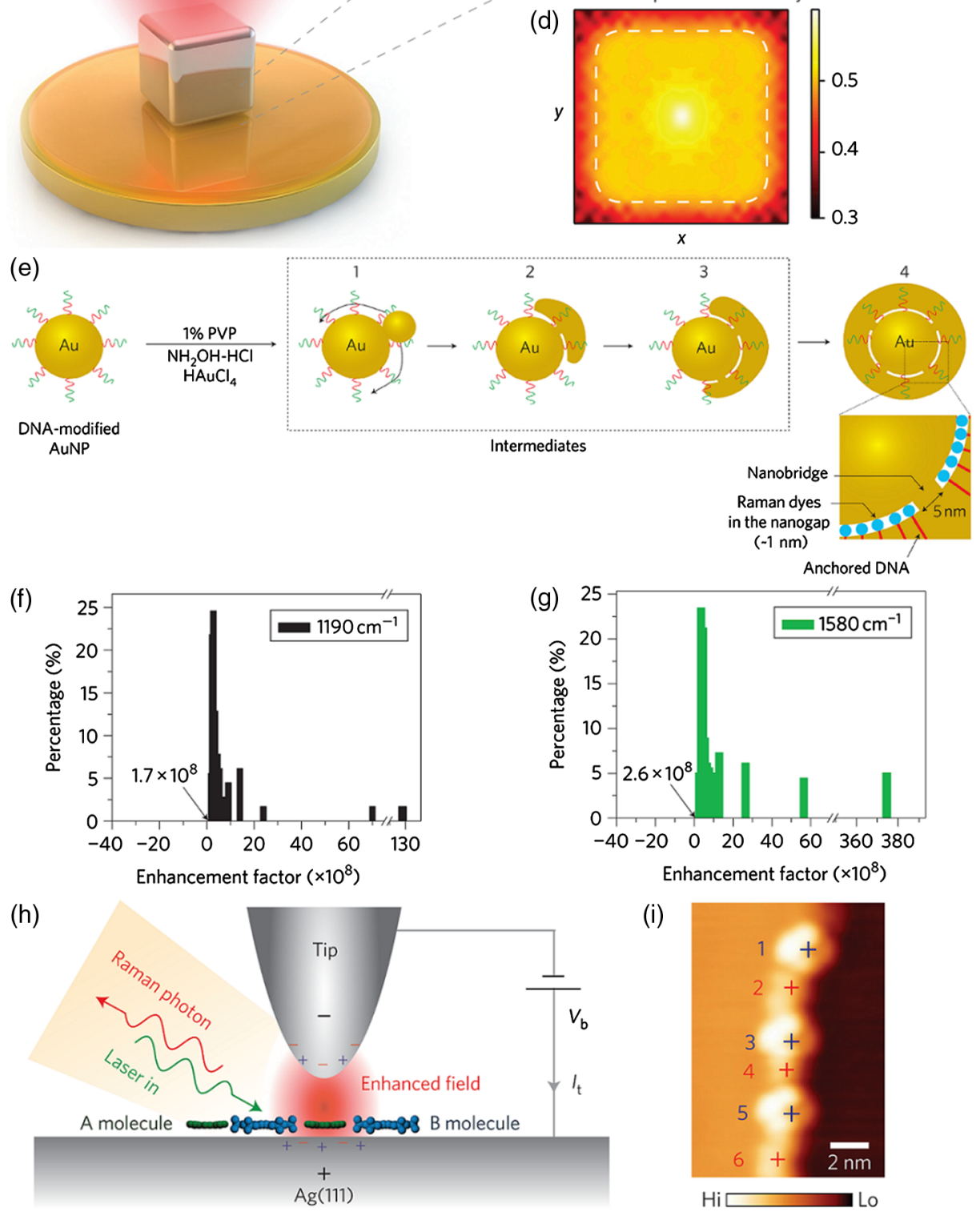

Fig. 2 (a) Schematic of a silver nanocube on a gold film separated by a layer of fluorescent material. The red cone indicates the enhanced emission from the system. (b) Transmission electron microscopy image of a single silver nanocube. Scale bar, $50 \mathrm{~nm}$. (c) Schematic cross section of the system with maximum field enhancement of $\sim 100$ in the gap. (d) Map of the enhanced quantum efficiency of the dipoles as a function of position under the nanocube. Reproduced with permission from Ref. 30. (e) Synthesis scheme for DNA-modified Au-NNPs. (f) and (g) Distribution of measured electromagnetic enhancement factors at 1190 and $1580 \mathrm{~cm}^{-1}$, respectively. Reproduced with permission from Ref. 31. (h) Schematic of STM-controlled TERS on Ag (111) with an Ag tip. (I) STM map of a mixed molecular chain self-assembled at the step edge of $\mathrm{Ag}$ (111). Reproduced with permission from Ref. 32. 


\section{Breaking the Diffusion Limit of Single Molecules by Increasing the Molecular Concentration}

Engineering of the near-field excitation light with plasmatic nanostructures allows for the control of single-molecule emissions at the nanoscale. However, this will not guarantee the successful detection of single molecules in practice, especially when the molecules are in a highly diluted solution with concentrations in the femtomolar $\left(10^{-15}\right)$ or attomolar $\left(10^{-18}\right)$ range. This is mainly because it takes an unrealistically long time for the molecules to diffuse to the small detection area, or in other words, it is diffusion-limited. ${ }^{53-57}$ To break this diffusion limit, several methods have been demonstrated that allow for the significant reduction of the single-molecule diffusion time by increasing the concentration of the molecular solutions in the detection area.

De Angelis et al. ${ }^{56}$ have demonstrated that a few molecules can be localized and detected at an attomolar concentration by combining superhydrophobic artificial surfaces and plasmonic nanostructures. The basic idea is schematically shown in Fig. 3(a) as follows: (1) put a drop of a solution that contains the molecules on a hydrophobic surface; (2) let the droplet evaporate to increase the concentration of the molecules in the drop; (3) as the concentration increases, the detection time decreases until the molecule eventually sticks on the detection surface; and (4) the detection surface is constructed using SERS active structures so that the single molecule can be detected with SERS. In this work, the hydrophobic surface is made of periodical silicon micropillar arrays as shown in the SEM image of Fig. 3(b). The hydrophobic surface allows the droplet to slide on the surface and, at the same time, avoids it being pinned at its initial contact point. ${ }^{58-61}$ Figure 3(c) shows the measurement of the contact angle of the droplet during the evaporation at four different times, which is almost constant. The recognition and localization of a single lambda DNA molecule has been successfully demonstrated using this system. Recently, Wong's group ${ }^{62}$ developed a slippery substrate that allows for the free movement of the droplet on a substrate to achieve similar functions.

To break the diffusion limit, the solution can also be forced to flow through nanochannels to increase the molecular concentration in the detection area. ${ }^{63,64}$ Figure 4(a) schematically shows the working mechanism of such a system. ${ }^{63}$ An array of nanoholes in a gold film is integrated with a microfluidic system to serve as both a plasmonic sensor and flow-through nanochannels. A voltage is applied across the solution to force the solution to flow through the nanochannels and concentrate the analyte on the plasmonic sensor. Figure 4(b) shows the evolution of the fluorescence signal collected from the nanoholes by using this method. The increase in

(a)



(c)

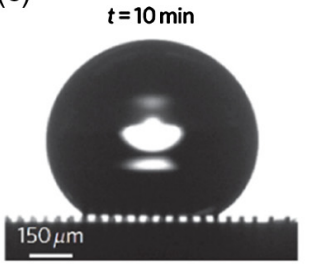

(b)



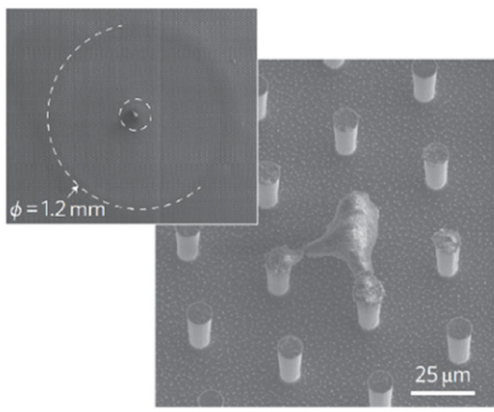

$t=25 \min$
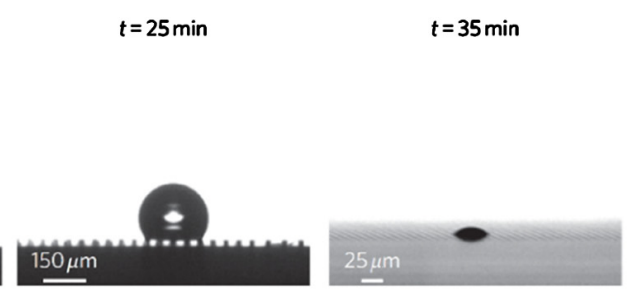

Fig. 3 (a) Schematic of the working principle of the single-molecule detection beyond the diffusion limit. (b) SEM images of the sample after the evaporation of the sample solution. (c) Contact angle and droplet size changes during evaporation at four different times. Reproduced with permission from Ref. 56. 


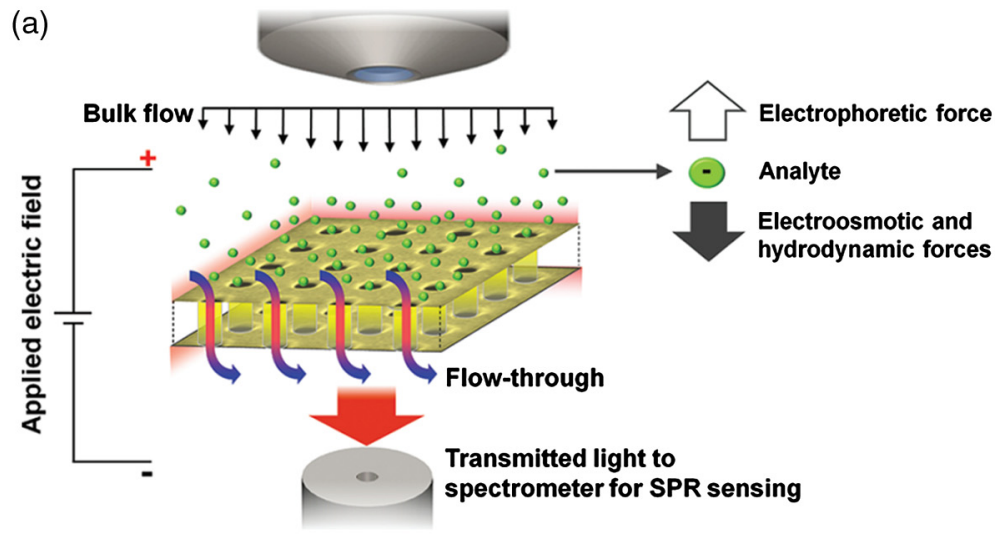

(b)

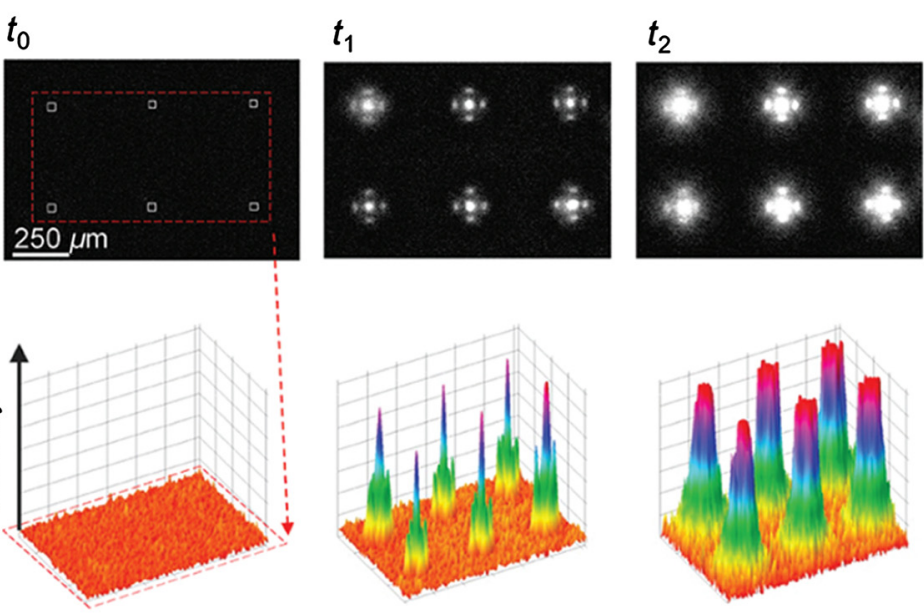

Fig. 4 (a) Mechanism of flow-through sensing through nanochannels. (b) Image sequence showing the progress of fluorescein concentration at the nanochannels (top panel) and their corresponding surface plots. Reproduced with permission from Ref. 63.

the concentration of the analytes on the sensor is clearly demonstrated. In addition to these techniques, other methods have also been developed to increase the binding probability of the analytes on the sensor and to increase the detection speed. For example, the surfaces of plasmonic nanoparticles serving as sensors can be antibody modified to target the molecules, which is another effective way to increase the binding rate and reduce the detection time. ${ }^{65-70}$

\section{Enhanced Single-Molecule Detection at Physiological Concentrations Using Optical Aperture Nanoantennas}

Another detection that seems easy but is very challenging happens at native physiological concentrations. Many biologically relevant processes occur at high concentrations, for example, micromolar $\left(10^{-6}\right)$ concentrations. Therefore, single-molecule detection at these high concentrations is highly desired. ${ }^{5,6}$ A common sense is that the higher the concentration of the molecules, the higher the signal and the easier the detection. However, the problem is that it is difficult to distinguish the signal from a single molecule at such high concentrations. The higher the concentrations, the smaller the average distance between adjacent molecules. Therefore, the distance between two molecules will be much smaller than the diffraction limit of light, which means it is almost impossible to excite only one molecule at a time with diffraction-limited light except that the molecule itself can be photo activatable and separated temporally like the molecules used in super-resolution imaging. ${ }^{71,72}$

The ODN introduced in Sec. 2 can be used to focus light at the nanoscale and excites only one molecule at a time at a high concentration. ${ }^{37,38,73,74}$ However, the ODNs are typically illuminated with diffraction-limited light; therefore, the molecules are not only excited by the near-field light of the ODNs but also by the diffraction-limited light. The fluorescence signal 
from the molecules excited by the diffraction-limited light may contribute a background noise to the detection. Optical aperture nanoantennas (OANs), which consist of nanoapertures formed on a metallic film, have been developed to overcome this limitation. ${ }^{8,9,75,76}$ The OANs can effectively block the incident light and confine the excitation light in the nanoaperture. Therefore, it can excite only one molecule at a time and gives near-zero background noise. The OANs with these properties are ideal for single-molecule detection at high concentrations. Zhao's group has provided a review on this topic before, and we refer the reader to Ref. 8 for more details. In this section, we provide a brief and updated overview on this topic.

A commonly used OAN is a circular nanohole that is formed in a metallic film such as a gold film. Djaker et al. ${ }^{77}$ have investigated the SERS of 4-aminothiophenol (pMA) with such an OAN. The pMA is self-assembled inside the OAN. An average SERS enhancement factor of 250 is obtained when the aperture size is $100 \mathrm{~nm}$ because of the effects of SPR on the aperture. OANs with other designs have been demonstrated such as the one shown in Fig. $5(\mathrm{a}){ }^{78}$ The OAN consists of two touching nanoholes to form a double-hole shaped OAN, which can give a large field enhancement at the apex between the holes when the polarization of the exciting light is parallel to the apex as shown in Figs. 5(b) and 5(c). Gordon's group has systematically investigated the optical properties of this double-hole shaped OAN. ${ }^{80-82}$ Figure 5(d) shows the fluorescence correlation spectroscopy analysis and the corresponding temporal correlation of the intensity traces, which illustrates a reduced number of molecules within the nanoscale detection volume when the excitation is set parallel to the apex region. The confocal measurement for the reference solution demonstrates a reasonably high average fluorescence intensity as shown by the green curve in Fig. 5(b) ${ }^{78}$ The same group later showed that this double-hole shaped OAN can also be used for trapping and sensing single proteins. ${ }^{79}$ Figure 5(e) shows the real-time optical transmission signal from the OAN when different agents are trapped and sensed by the OAN. The signal at steps (a), (b), and (c) in Fig. 5(e) shows the optical signal without a trap, with a 20-nm biotin-coated polystyrene (PS) particle trapped, and binding of streptavidin with the trapped biotin-coated PS particle, respectively.

The OANs formed on a gold film as shown in Fig. 5 can block the light outside the aperture; however, some light can still reach the top surface due to plasmonic coupling through the

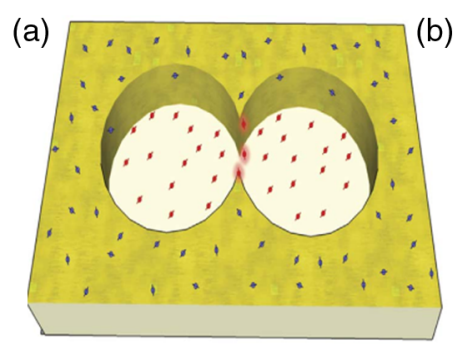

(d)

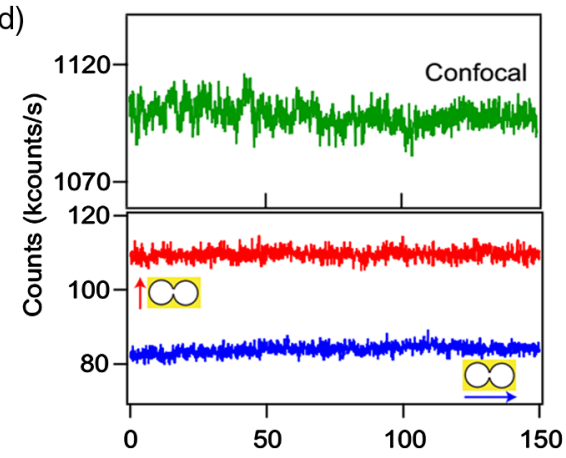

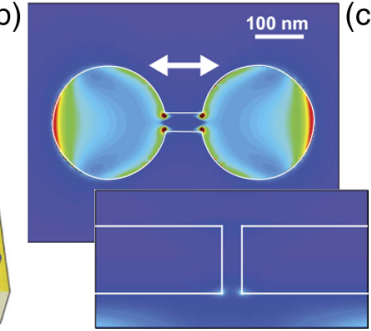

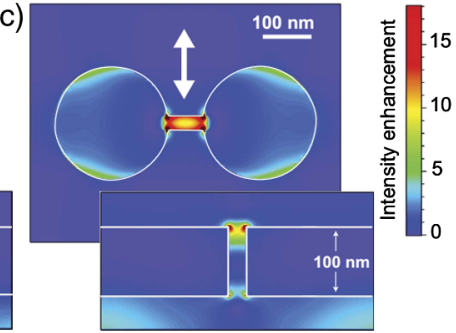

(e)

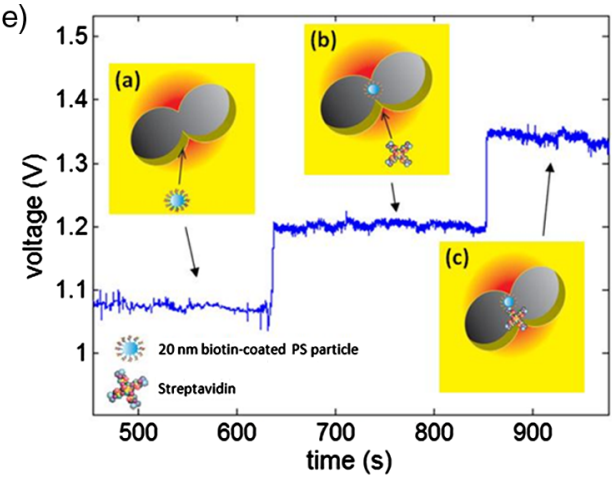

Fig. 5 (a) Sketch of a double-hole shaped OAN to enhance single-molecule fluorescence. (b, c) Intensity enhancement of the double-hole shaped OAN (25-nm gap and 190-nm diameter excited at $633 \mathrm{~nm}$ ) with a polarization perpendicular (b) and parallel (c) to the apex between the holes. (d) Fluorescence time trace with excitation light parallel (red line) and perpendicular (blue line) to the apex. The green line shows the time trace measured in the confocal case for comparison. (e) Time trace of optical transmission signal from the double-hole shaped OAN for trapping and sensing single proteins. Reproduced with permission from Refs. 78 and 79. 
aperture. ${ }^{83}$ The "leaked" light to the top surface will excite extra molecules that may contribute a background noise to single-molecule detection at high concentrations. An ideal OAN for singlemolecule detection at high concentrations should focus the light only at the bottom of the aperture with minimum light on the top surface. OANs that are formed on hybrid metallic film, such as $\mathrm{Al}-\mathrm{Au}^{83}$ and $\mathrm{Cr}-\mathrm{Au}^{84}$ films, have been used to achieve this goal. The top metallic film allows for the quenching of the top field on the Au film, therefore confining the light more efficiently at the bottom of the aperture.

Limited by the current nanofabrication technologies, the nanoaperture in an OAN is typically larger than the nanogap in an ODN. Therefore, the ODN gives rise to a larger field enhancement than the OAN. Wenger's group has designed a so-called antenna-in-box, ${ }^{85,86}$ which is formed by fabricating an ODN inside an OAN. This design combines the advantages of the OAN's low background noise with the ODN's high field enhancement. The nanogap in the ODN in the antenna-in-box can be fabricated with a much smaller size than the nanoaperture in an OAN. Recently, Flauraud et al. ${ }^{87}$ have developed a nanofabrication technique that allows for the
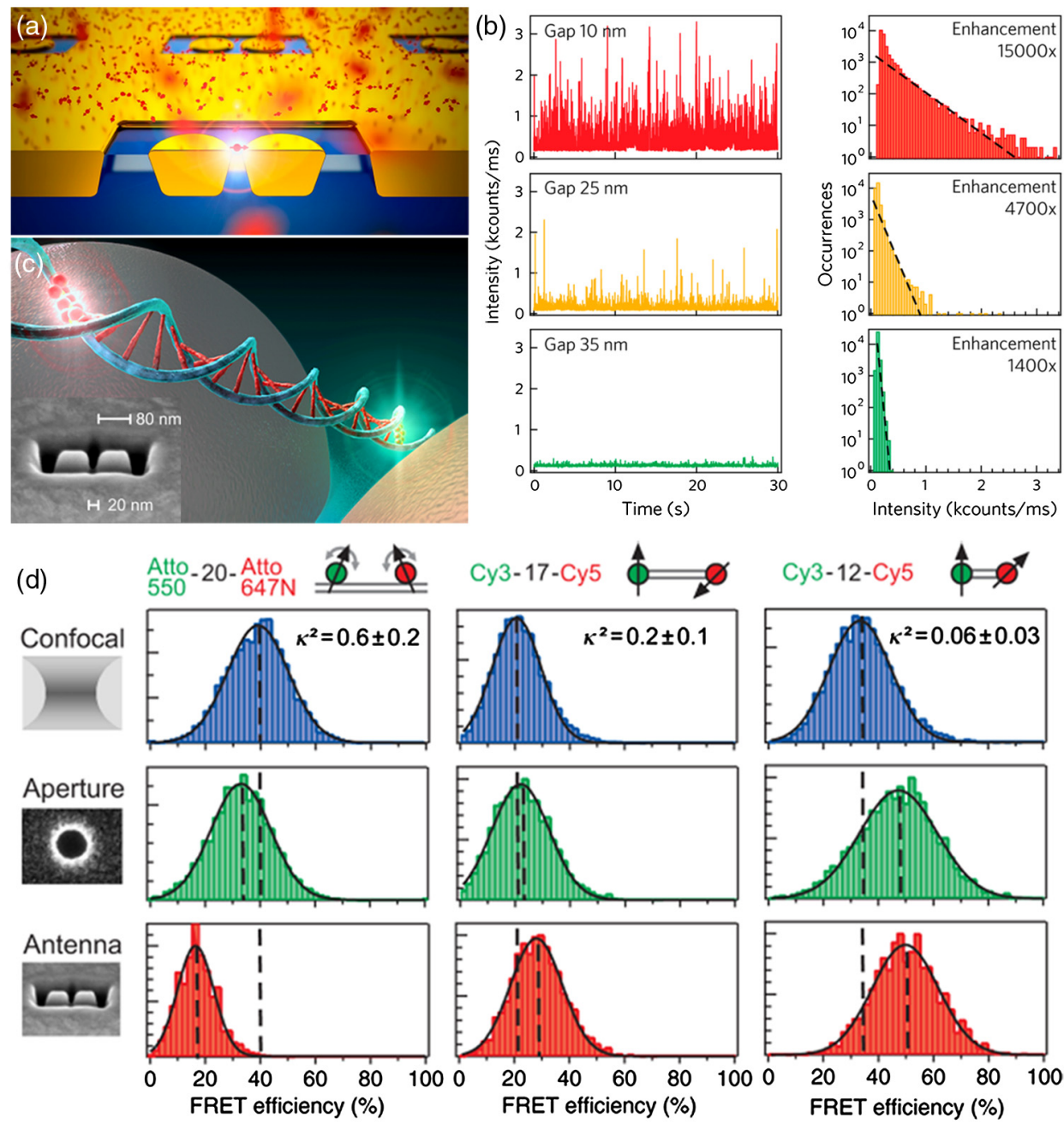

Fig. 6 (a) Schematic of an antenna-in-box array. (b) FE enhancement of crystal violet molecules on antenna-in-boxes with different gap sizes. The left column is the fluorescence time traces. The right column shows the corresponding photon count rate histograms. Reproduced with permission from Ref. 87. (c) Schematic of an antenna-in-box for the enhancement of FRET efficiency. Inset shows the scanning electron microscope image of an antenna-in-box that is fabricated in an aluminum film. (d) Comparison of the FRET efficiency histograms for different configurations: confocal (first row), 200-nm diameter aperture (second row), and antenna-in-box with 20-nm gap (third row). $\kappa^{2}$, which is the orientation parameter, describes the relative orientation between the donor and acceptor transition dipoles. Reproduced with permission from Ref. 88. 
fabrication of large flat arrays of such antenna-in-boxes featuring $10 \mathrm{~nm}$ gaps as schematically shown in Fig. 6(a). Figure 6(b) shows the FE of crystal violet molecules from the nanoantenna with different gaps. Such an antenna-in-box can be used to enhance single-molecule fluorescence by a factor of $10^{4}$ to $10^{5}$ and to reduce the detection volumes to 20 zeptoliter. The antenna-in-box can also be used to enhance the FRET efficiency as schematically shown in Fig. 6(c). Torres et al. ${ }^{88}$ have demonstrated that the strong inhomogeneous and localized electric field in the antenna-in-box opens new energy transfer routes; therefore, it overcomes the limitations from the mutual dipole orientation in conventional FRET and ultimately enhances the FRET efficiency. It also enables the energy transfer between near-perpendicular orientations of the donor and acceptor dipoles that would be otherwise forbidden in conventional FRET. Figure 6(d) compares the FRET efficiency histograms for different nanostructures that are in confocal setup, 200-nm diameter aperture, and antenna-in-box with 20-nm gap antenna. FRET efficiency at different configurations of the donor and acceptor, which is defined by the orientation parameter $\kappa^{2}$, is also investigated. $\kappa^{2}$ has a maximum value of four when both dipoles are aligned, whereas it is zero for dipoles in parallel planes with perpendicular orientation. The FRET efficiency increased $>50 \%$ for the antenna-in-box with the smallest orientation parameter $\left(\kappa^{2}=0.06\right)$ for the Cy3-12-Cy5 sample.

\section{Perspectives}

We have witnessed the rapid development of this promising field in recent years. Engineering of the excitation light and the solution of molecules allows for the enhancement of single-molecule detection at both ultralow and physiological concentrations. In addition, the radiation of the molecules can be controlled by the plasmonic nanostructures. Based on these recent developments, we provide insight into some new possibilities for future developments in this promising field.

\subsection{Broadband and Near-Infrared Operation}

Most plasmonic nanostructures are designed to have a narrow resonance spectrum such as at the excitation or emission spectrum of a molecule, but not both. However, it is highly desired to optimize the SPR of the optical nanoantennas so that it is optimized for both the excitation and the emission spectrum of a molecule to achieve maximum enhancement of its fluorescence. Recently, Liu and Lei ${ }^{89}$ proposed a double-resonant AuNR that can be used to match both the excitation and emission wavelengths of an emitter. In addition, optical detection of single molecules in the near-infrared window would be beneficial for many bioapplications because of the deep light penetration and reduced background absorption for this spectrum..$^{90,91}$

\subsection{Multiplex Detection}

There is increasing interest and demand in the simultaneous detection of multiple molecular targets for many biomedical applications. Optical detection based on fluorescence and Raman signals can both be used for this purpose. While the fluorescence method requires multiple labeling of the target molecules for multiplex detection, ${ }^{92,93}$ Raman has its intrinsic advantages in terms of multiplicity and sensitivity. For example, multiplex detection of viral antigens, ${ }^{94}$ bacterial meningitis, ${ }^{95}$ and cancer markers ${ }^{96}$ using SERS has been demonstrated. In addition, the combination of fluorescence, Raman, and plasmonic nanostructures on the same platform will allow for multiple and parallel detection of molecular targets as well as reduced detection times. ${ }^{97,98}$

\subsection{Miniaturization}

The engineering of the optical field with far-field optics typically involves many bulk optical elements, which occupy a large physical space. In contrast, the engineering of the near-field light with plasmonic nanostructures can reduce the system size significantly and possibly integrate the whole system on a single chip. However, bulk optics together with free-space lasers are still used to illuminate the plasmonic nanostructures in most of the single-molecule detection systems, which limits the further miniaturization of the system. Light sources with small form factors 
are desperately needed for these systems. Integrated silicon photonic devices with plasmonic nanostructures are a possible way to overcome this limitation. Silicon photonic devices have undergone a rapid development in recent years. ${ }^{99-102}$ Their fabrication shares the commercially complementary metal-oxide semiconductor chip fabrication facilities and, therefore, allows for low-cost and massive production. In addition, sample solution handling with microfluidic technology and imaging with smartphones ${ }^{103}$ will further facilitate the miniaturization systems for single-molecule detection.

\subsection{Heat Reduction}

The plasmonic nanostructures for single-molecule detection are typically made of metallic materials. Upon light illumination, heat is generated around these nanostructures due to the intrinsic ohmic loss in the materials. The heat generation disturbs the fluid flow in the system and may cause unpredictable effects for the single-molecule detection, which itself is an interesting topic to study. Therefore, the heat generation must be minimized in these systems. Caldarola et al. ${ }^{104}$ have proposed to use a nonplasmonic platform based on dielectric nanostructures to reduce the heat generation, which may be beneficial for new developments in this field.

\subsection{Detection-Time Reduction}

The natural evaporation of the sample solution has been applied to break the diffusion limit in single-molecule detection. ${ }^{56,62}$ However, this process still takes several minutes. New technologies are required to further reduce the detection time. This might be achieved by either reducing the droplet size with microfluidic devices or by speeding up the droplet evaporation process.

\section{Acknowledgments}

The authors acknowledge the financial support from the Hanley Sustainability Institute (HSI), the Summer Research Fellowship, and the Graduate Student Summer Fellowship at the University of Dayton. The authors declare no conflict of interest.

\section{References}

1. M. Pitschke et al., "Detection of single amyloid $\beta$-protein aggregates in the cerebrospinal fluid of Alzheimer's patients by fluorescence correlation spectroscopy," Nat. Med. 4(7), 832-834 (1998).

2. J. Eid et al., "Real-time DNA sequencing from single polymerase molecules," Science 323(5910), 133-138 (2009).

3. S. Uemura et al., "Real-time tRNA transit on single translating ribosomes at codon resolution," Nature 464(7291), 1012-1017 (2010).

4. K. Bacia, S. A. Kim, and P. Schwille, "Fluorescence cross-correlation spectroscopy in living cells," Nat. Methods 3(2), 83-89 (2006).

5. K. T. Samiee et al., " $\lambda$-repressor oligomerization kinetics at high concentrations using fluorescence correlation spectroscopy in zero-mode waveguides," Biophys. J. 88(3), 2145-2153 (2005).

6. J. M. Moran-Mirabal and H. G. Craighead, "Zero-mode waveguides: sub-wavelength nanostructures for single molecule studies at high concentrations," Methods 46(1), 11-17 (2008).

7. J. Wenger and H. Rigneault, "Photonic methods to enhance fluorescence correlation spectroscopy and single molecule fluorescence detection," Int. J. Mol. Sci. 12(1), 206-221 (2010).

8. M. S. Alam, F. Karim, and C. Zhao, "Single-molecule detection at high concentrations with optical aperture nanoantennas," Nanoscale 8(18), 9480-9487 (2016).

9. D. Punj et al., "Plasmonic antennas and zero-mode waveguides to enhance single molecule fluorescence detection and fluorescence correlation spectroscopy toward physiological concentrations," Wiley Interdiscip. Rev. Nanomed. Nanobiotechnol. 6(3), 268-282 (2014). 
10. A. M. Kern et al., "Enhanced single-molecule spectroscopy in highly confined optical fields: from $\lambda / 2$-Fabry-Pérot resonators to plasmonic nano-antennas," Chem. Soc. Rev. 43(4), 1263-1286 (2014).

11. P. Holzmeister et al., "Breaking the concentration limit of optical single-molecule detection," Chem. Soc. Rev. 43(4), 1014-1028 (2014).

12. E. Di Fabrizio et al., "Roadmap on biosensing and photonics with advanced nano-optical methods," J. Opt. 18(6), 063003 (2016).

13. D. K. Gramotnev and S. I. Bozhevolnyi, "Plasmonics beyond the diffraction limit," Nat. Photonics 4(2), 83-91 (2010).

14. J. A. Schuller et al., "Plasmonics for extreme light concentration and manipulation," Nat. Mater. 9(3), 193-204 (2010).

15. X. Ma et al., "Noble-metal-free plasmonic photocatalyst: hydrogen doped semiconductors," Sci. Rep. 4, 3986 (2014).

16. S. S. K. Guduru et al., "Plasmonic heavily-doped semiconductor nanocrystal dielectrics: making static photonic crystals dynamic," J. Phys. Chem. C 119(5), 2775-2782 (2015).

17. Z. Wu et al., "Tunable graphene metasurfaces with gradient features by self-assemblybased moiré nanosphere lithography," Adv. Opt. Mater. 4(12), 2035-2043 (2016).

18. A.N. Grigorenko, M. Polini, and K. S. Novoselov, "Graphene plasmonics," Nat. Photonics 6, 749-758 (2012).

19. S. A. Maier and H. A. Atwater, "Plasmonics: localization and guiding of electromagnetic energy in metal/dielectric structures," J. Appl. Phys. 98(1), 011101 (2005).

20. P. Zijlstra, P. M. R. Paulo, and M. Orrit, "Optical detection of single non-absorbing molecules using the surface plasmon resonance of a gold nanorod," Nat. Nanotechnol. 7(6), 379-382 (2012).

21. W. L. Barnes, A. Dereux, and T. W. Ebbesen, "Surface plasmon subwavelength optics," Nature 424(6950), 824-830 (2003).

22. J. Wenger et al., "Emission and excitation contributions to enhanced single molecule fluorescence by gold nanometric apertures," Opt. Express 16(5), 3008-3020 (2008).

23. P. Bharadwaj and L. Novotny, "Spectral dependence of single molecule fluorescence enhancement," Opt. Express 15(21), 14266-14274 (2007).

24. E. Wientjes et al., "Strong antenna-enhanced fluorescence of a single light-harvesting complex shows photon antibunching," Nat. Commun. 5, 4236 (2014).

25. A. Kinkhabwala et al., "Large single-molecule fluorescence enhancements produced by a bowtie nanoantenna," Nat. Photonics 3(11), 654-657 (2009).

26. N. Liu et al., "Nanoantenna-enhanced gas sensing in a single tailored nanofocus," Nat. Mater. 10(8), 631-636 (2011).

27. L. C. Estrada, P. F. Aramendía, and O. E. Martínez, "10000 times volume reduction for fluorescence correlation spectroscopy using nano-antennas," Opt. Express 16(25), 2059720602 (2008).

28. J. B. Khurgin, G. Sun, and R. A. Soref, "Practical limits of absorption enhancement near metal nanoparticles," Appl. Phys. Lett. 94(7), 071103 (2009).

29. G. Sun, J. B. Khurgin, and R. A. Soref, "Practical enhancement of photoluminescence by metal nanoparticles," Appl. Phys. Lett. 94(10), 101103 (2009).

30. G. M. Akselrod et al., "Probing the mechanisms of large Purcell enhancement in plasmonic nanoantennas," Nat. Photonics 8(11), 835-840 (2014).

31. D. K. Lim et al., "Highly uniform and reproducible surface-enhanced Raman scattering from DNA-tailorable nanoparticles with 1-nm interior gap," Nat. Nanotechnol. 6(7), $452-$ 460 (2011).

32. S. Jiang et al., "Distinguishing adjacent molecules on a surface using plasmon-enhanced Raman scattering," Nat. Nanotechnol. 10(10), 865-869 (2015).

33. J. B. Lassiter et al., "Plasmonic waveguide modes of film-coupled metallic nanocubes," Nano Lett. 13(12), 5866-5872 (2013).

34. A. Moreau et al., "Controlled-reflectance surfaces with film-coupled colloidal nanoantennas," Nature 492(7427), 86-89 (2012).

35. A. Rose et al., "Control of radiative processes using tunable plasmonic nanopatch antennas," Nano Lett. 14(8), 4797-4802 (2014). 
36. M. Yi et al., "Plasmonic interaction between silver nano-cubes and a silver ground plane studied by surface-enhanced Raman scattering," Plasmonics 6(3), 515-519 (2011).

37. G. P. Acuna et al., "Fluorescence enhancement at docking sites of DNA-directed selfassembled nanoantennas," Science 338(6106), 506-510 (2012).

38. A. Puchkova et al., "DNA origami nanoantennas with over 5000 -fold fluorescence enhancement and single-molecule detection at $25 \mu \mathrm{M}$," Nano Lett. 15(12), 8354-8359 (2015).

39. V. V. Thacker et al., "DNA origami based assembly of gold nanoparticle dimers for surface-enhanced Raman scattering," Nat. Commun. 5, 3448 (2014).

40. P. Kühler et al., "Plasmonic DNA-origami nanoantennas for surface-enhanced Raman spectroscopy," Nano Lett. 14(5), 2914-2919 (2014).

41. J. Prinz et al., "DNA origami based Au-Ag-core-shell nanoparticle dimers with singlemolecule SERS sensitivity," Nanoscale 8(10), 5612-5620 (2016).

42. W. Li et al., "Dimers of silver nanospheres: facile synthesis and their use as hot spots for surface-enhanced Raman scattering," Nano Lett. 9(1), 485-490 (2009).

43. D. Graham et al., "Control of enhanced Raman scattering using a DNA-based assembly process of dye-coded nanoparticles," Nat. Nanotechnol. 3(9), 548-551 (2008).

44. W. H. Park and Z. H. Kim, "Charge transfer enhancement in the SERS of a single molecule," Nano Lett. 10(10), 4040-4048 (2010).

45. Y. Fang, N.H. Seong, and D. D. Dlott, "Measurement of the distribution of site enhancements in surface-enhanced Raman scattering," Science 321(5887), 388-392 (2008).

46. H. Im et al., "Vertically oriented sub-10-nm plasmonic nanogap arrays," Nano Lett. 10(6), 2231-2236 (2010).

47. M. S. Anderson, "Locally enhanced Raman spectroscopy with an atomic force microscope," Appl. Phys. Lett. 76(21), 3130-3132 (2000).

48. M. D. Sonntag et al., "Recent advances in tip-enhanced Raman spectroscopy," J. Phys. Chem. Lett. 5(18), 3125-3130 (2014).

49. R. Zhang et al., "Chemical mapping of a single molecule by plasmon-enhanced Raman scattering," Nature 498(7452), 82-86 (2013).

50. J. Zhang, Y. Fu, and J. R. Lakowicz, "Enhanced Förster resonance energy transfer (FRET) on a single metal particle," J. Phys. Chem. C 111(1), 50-56 (2007).

51. C. S. Yun et al., "Nanometal surface energy transfer in optical rulers, breaking the FRET barrier," J. Am. Chem. Soc. 127(9), 3115-3119 (2005).

52. M. Wang et al., "Molecular-fluorescence enhancement via blue-shifted plasmon-induced resonance energy transfer," J. Phys. Chem. C 120(27), 14820-14827 (2016).

53. P. E. Sheehan and L. J. Whitman, "Detection limits for nanoscale biosensors," Nano Lett. 5(4), 803-807 (2005).

54. P. R. Nair and M. A. Alam, "Performance limits of nanobiosensors," Appl. Phys. Lett. 88(23), 233120 (2006).

55. P. R. Nair and M. A. Alam, "Screening-limited response of nanobiosensors," Nano Lett. 8(5), 1281-1285 (2008).

56. F. De Angelis et al., "Breaking the diffusion limit with super-hydrophobic delivery of molecules to plasmonic nanofocusing SERS structures," Nat. Photonics 5(11), 682-687 (2011).

57. T. M. Squires, R. J. Messinger, and S. R. Manalis, "Making it stick: convection, reaction and diffusion in surface-based biosensors," Nat. Biotechnol. 26(4), 417-426 (2008).

58. G. McHale, N. J. Shirtcliffe, and M. I. Newton, "Super-hydrophobic and super-wetting surfaces: analytical potential?," Analyst 129(4), 284-287 (2004).

59. L. Mahadevan and Y. Pomeau, "Rolling droplets," Phys. Fluids 12(9), 2449-2453 (1999).

60. P. Aussillous and D. Quéré, "Liquid marbles," Nature 411(6840), 924-927 (2001).

61. G. McHale et al., "Analysis of droplet evaporation on a superhydrophobic surface," Langmuir 21(24), 11053-11060 (2005).

62. S. Yang et al., "Ultrasensitive surface-enhanced Raman scattering detection in common fluids," Proc. Natl. Acad. Sci. U. S. A. 113(2), 268-273 (2016).

63. C. Escobedo et al., "Optofluidic concentration: plasmonic nanostructure as concentrator and sensor," Nano Lett. 12(3), 1592-1596 (2012). 
64. F. Eftekhari et al., "Nanoholes as nanochannels: flow-through plasmonic sensing," Anal. Chem. 81(11), 4308-4311 (2009).

65. D. S. Grubisha et al., "Femtomolar detection of prostate-specific antigen: an immunoassay based on surface-enhanced Raman scattering and immunogold labels," Anal. Chem. 75(21), 5936-5943 (2003).

66. A. Lesniewski et al., "Antibody modified gold nanoparticles for fast and selective, colorimetric T7 bacteriophage detection," Bioconjug. Chem. 25(4), 644-648 (2014).

67. Y. Wang and E. C. Alocilja, "Gold nanoparticle-labeled biosensor for rapid and sensitive detection of bacterial pathogens," J. Biol. Eng. 9, 16 (2015).

68. J. N. Anker et al., "Biosensing with plasmonic nanosensors," Nat. Mater. 7(6), 442-453 (2008).

69. B. Malile and J. I. L. Chen, "Morphology-based plasmonic nanoparticle sensors: controlling etching kinetics with target-responsive permeability gate," J. Am. Chem. Soc. 135(43), 16042-16045 (2013).

70. S. Unser et al., "Localized surface plasmon resonance biosensing: current challenges and approaches," Sensors 15(7), 15684-15716 (2015).

71. M. J. Rust, M. Bates, and X. Zhuang, "Sub-diffraction-limit imaging by stochastic optical reconstruction microscopy (STORM)," Nat. Methods 3(10), 793-796 (2006).

72. E. Betzig et al., "Imaging intracellular fluorescent proteins at nanometer resolution," Science 313(5793), 1642-1645 (2006).

73. S. Khatua, H. Yuan, and M. Orrit, "Enhanced-fluorescence correlation spectroscopy at micro-molar dye concentration around a single gold nanorod," Phys. Chem. Chem. Phys. 17(33), 21127-21132 (2015).

74. Q. Yuan et al., "Targeted bioimaging and photodynamic therapy nanoplatform using an aptamer-guided G-quadruplex DNA carrier and near-infrared light," Angew. Chem. Int. Ed. 52(52), 13965-13969 (2013).

75. D. R. Walt, "Optical methods for single molecule detection and analysis," Anal. Chem. 85(3), 1258-1263 (2013).

76. P. Holzmeister et al., "Breaking the concentration limit of optical single-molecule detection," Chem. Soc. Rev. 43(4), 1014-1028 (2014).

77. N. Djaker et al., "Surface enhanced Raman scattering on a single nanometric aperture," J. Phys. Chem. C 114(39), 16250-16256 (2010).

78. R. Regmi et al., "Nanoscale volume confinement and fluorescence enhancement with double nanohole aperture," Sci. Rep. 5, 15852 (2015).

79. A. A. Al Balushi, A. Zehtabi-Oskuie, and R. Gordon, "Observing single protein binding by optical transmission through a double nanohole aperture in a metal film," Biomed. Opt. Express 4(9), 1504-1511 (2013).

80. A. Lesuffleur, L. K. S. Kumar, and R. Gordon, "Enhanced second harmonic generation from nanoscale double-hole arrays in a gold film," Appl. Phys. Lett. 88, 261104 (2006).

81. Q. Min et al., "Localized Raman enhancement from a double-hole nanostructure in a metal film," J. Phys. Chem. C 112(39), 15098-15101 (2008).

82. L. K. S. Kumar et al., "Double nanohole apex-enhanced transmission in metal films," Appl. Phys. B 84, 25-28 (2006).

83. C. Zhao et al., "Single-molecule detection and radiation control in solutions at high concentrations via a heterogeneous optical slot antenna," Nanoscale 6(15), 9103-9109 (2014).

84. H. Aouani et al., "Plasmonic antennas for directional sorting of fluorescence emission," Nano Lett. 12(6), 2400-2406 (2011).

85. P. Ghenuche et al., "Matching nanoantenna field confinement to FRET distances enhances förster energy transfer rates," Nano Lett. 15(9), 6193-6201 (2015).

86. D. Punj et al., "A plasmonic 'antenna-in-box' platform for enhanced single-molecule analysis at micromolar concentrations," Nat. Nanotechnol. 8(7), 512-516 (2013).

87. V. Flauraud et al., "In-plane plasmonic antenna arrays with surface nanogaps for giant fluorescence enhancement," Nano Lett. 17(3), 1703-1710 (2017).

88. J. de Torres et al., "Plasmonic nanoantennas enable forbidden Förster dipole-dipole energy transfer and enhance the FRET efficiency," Nano Lett. 16(10), 6222-6230 (2016). 
89. X. Liu and D. Y. Lei, "Simultaneous excitation and emission enhancements in upconversion luminescence using plasmonic double-resonant gold nanorods," Sci. Rep. 5, 15235 (2015).

90. G. Hong, A. L. Antaris, and H. Dai, "Near-infrared fluorophores for biomedical imaging," Nat. Biomed. Eng. 1, 10 (2017).

91. S. A. Hilderbrand and R. Weissleder, "Near-infrared fluorescence: application to in vivo molecular imaging," Curr. Opin. Chem. Biol. 14(1), 71-79 (2010).

92. F. Ma et al., "Fluorescent biosensors based on single-molecule counting," Acc. Chem. Res. 49(9), 1722-1730 (2016).

93. F. Ma et al., "Multiplex detection of histone-modifying enzymes by total internal reflection fluorescence-based single-molecule detection," Chem. Coтmun. 52(6), 1218-1221 (2016).

94. J. Neng et al., "Surface-enhanced Raman scattering (SERS) detection of multiple viral antigens using magnetic capture of SERS-active nanoparticles," Biosens. Bioelectron. 41, 316-321 (2013).

95. K. Gracie et al., "Simultaneous detection and quantification of three bacterial meningitis pathogens by SERS," Chem. Sci. 5(3), 1030-1040 (2014).

96. S. Ye et al., "Asymmetric signal amplification for simultaneous SERS detection of multiple cancer markers with significantly different levels," Anal. Chem. 87(16), 8242-8249 (2015).

97. Y. Kim et al., "Simultaneous detection of EGFR and VEGF in colorectal cancer using fluorescence-Raman endoscopy," Sci. Rep. 7(1), 1035 (2017).

98. S. Jeong et al., "Fluorescence-Raman dual modal endoscopic system for multiplexed molecular diagnostics," Sci. Rep. 5, 9455 (2015).

99. N. C. Harris et al., "Efficient, compact and low loss thermo-optic phase shifter in silicon," Opt. Express 22(9), 10487-10493 (2014).

100. J. S. Levy et al., "CMOS-compatible multiple-wavelength oscillator for on-chip optical interconnects," Nat. Photonics 4(1), 37-40 (2009).

101. L. Razzari et al., "CMOS-compatible integrated optical hyper-parametric oscillator," Nat. Photonics 4, 41-45 (2010).

102. H. Guan et al., "CMOS-compatible highly efficient polarization splitter and rotator based on a double-etched directional coupler," Opt. Express 22(3), 2489-2496 (2014).

103. Q. Wei et al., "Fluorescent imaging of single nanoparticles and viruses on a smart phone," ACS Nano 7(10), 9147-9155 (2013).

104. M. Caldarola et al., "Non-plasmonic nanoantennas for surface enhanced spectroscopies with ultra-low heat conversion," Nat. Commun. 6, 7915 (2015).

Chenglong Zhao received his PhD from Peking University, Beijing, China, in 2011. He is an assistant professor at the University of Dayton with a joint appointment in the Department of Physics and Department of Electro-Optics and Photonics. Then, he carried out postdoctoral research at Pennsylvania State University and the National Institute of Standards and Technology. He leads the Nano-Photonic and Nano-Manipulation Lab, which is dedicated to the development of cutting-edge nanotechnologies for applications in additive nanomanufacturing, single-molecule detection, and ultrasensitive biosensing.

Biographies for the other authors are not available. 